
\title{
28 Research Square \\ The role of the MTG in negative emotional processing in young adults with autistic traits: a fMRI task study
}

Yaxu Yu

Southwest University

Xiaoqin Wang

Southwest University

Jamie Ward

University of Sussex

Qiu Jiang ( $\square$ qiuj318@swu.edu.cn )

Southwest University https://orcid.org/0000-0003-0269-5910

\section{Research}

Keywords: autistic traits, negative emotion processing, middle frontal gyrus, task fMRI, mediation, generalized psychophysiological interaction

Posted Date: December 11th, 2019

DOI: https://doi.org/10.21203/rs.2.18547/v1

License: (9) This work is licensed under a Creative Commons Attribution 4.0 International License.

Read Full License 


\section{Abstract}

Individuals with autistic traits are those within the normal population who demonstrate social, communication, and cognitive impairments but who do not meet the clinical threshold for autism spectrum disorder (ASD). ASD is a pervasive neurodevelopmental disorder associated with deficits in high-level cognitive functions such as negative emotion processing and social cognitive processing, and people with autistic traits also have these impairments. Few previous studies have explored negative emotion processing in people with autistic traits using task-based functional magnetic resonance imaging (fMRI). In this study, we focused on the behaviors of a large sample $(\mathrm{N}=104)$ of Chinese individuals with different levels of autistic traits, who we tested using a popular questionnaire called the autism spectrum quotient (AQ). We then applied task fMRI to determine negative emotion processing and social cognition associated with differences in brain activation and functional connectivity. The results mainly showed significantly positive activation in the right middle temporal gyrus (MTG), and the right hippocampus gyrus $(\mathrm{HG})$ in multiple regression analysis. More importantly, mediation analysis showed that the right MTG suppresses the relationship between AQ (total) score and negative emotional processing, showing that a higher autistic trait score in individuals leads to a smaller difference between the rating of negative images and the neutral images. Furthermore, generalized psychophysiological interaction analyses (gPPI) analysis also suggested that the right MTG shows significant functional connectivity $(\mathrm{FC})$ with the left parahippocampal gyrus $(\mathrm{PHG})$, suggesting that individuals with autistic traits have high level of spontaneous negative emotion. According to these findings, we assumed that the MTG might have a specific effect on processing negative emotion in individuals with autistic traits related to weaker to social emotion ability. The emotion perception mechanisms in the MTG region may be affected by atypical negative emotion processing in other brain regions such as $\mathrm{HG}$ and PHG, during a negative emotion processing task with socially emotion-relevant information in individuals with autistic traits.

\section{Background}

Individuals with autistic traits can be found in the general population (S. Baron-Cohen et al. 2001; John N. Constantino and Todd 2003; John N Constantino and Todd 2005), and have characteristics similar to those of autism spectrum disorder (ASD) patients, such as deficits in emotion processing and social functioning, worse communication abilities, and repetitive behaviors (Wheelwright et al. 2010; Barrio 2017; A. P. Association 2013). These are ubiquitous traits that most people show at different levels (Sucksmith et al. 2011). Currently, it is assumed that negative emotion processing and social cognitive function difficulties in people with autistic traits might be due to alterations in brain activation and functional connectivity (FC) between brain regions. Previous neuroimaging studies have concentrated on measuring brain activity from various areas (Redcay and Courchesne 2005; Carper and Courchesne 2005; Barros-Loscertales et al. 2011; Damarla et al. 2010; Hirata et al. 2018; Ikeda et al. 2018) by examining voxel-wise or region of interest (ROI) functional connectivity (Gotts et al. 2012; Schipul et al. 2012; Mueller et al. 2013; von dem Hagen et al. 2013; Alaerts et al. 2014; Tyszka et al. 2014; Zhu et al. 2014) and 
individual differences (Grootscholten et al. 2018; St Pourcain et al. 2018; Jansen et al. 2018; Sasamoto et al. 2011), making it possible to investigate the underlying neural correlates of autistic spectrum disorder. Few studies have used extremely negative images to study participants' negative emotions in individuals with autistic traits using specialized tasked fMRI.

ASD studies using functional and anatomical connectivity measures have reached a growing consensus that negative emotion processing and social cognitive function impairments are linked to abnormalities in specific brain areas in addition to genetics (Wass 2011; Vissers et al. 2012; Tyszka et al. 2014; Alaerts et al. 2014; Mueller et al. 2013; Muskett et al. 2019; Kliemann et al. 2018; Ciaramidaro et al. 2018; New et al. 2010). Some studies have found that ASD patients are sensitive to negative emotions (Kliemann et al. 2018; Ciaramidaro et al. 2018; Samson et al. 2015; Eack et al. 2015; Bird and Cook 2013), while other studies have shown that ASD patients are more likely to ignore negative emotions (Deruelle et al. 2008; Santos et al. 2012; Dawson et al. 2004; Katarzyna et al. 2010; Pierce et al. 2011). These differing views on negative emotions may have resulted from their studies focusing on activation in different brain areas. Paakki's ASD study tested the difference between the ASD and control groups. The results showed that compared to controls, the right superior temporal sulcus, inferior and middle frontal gyri had significantly decreased ReHo in the ASD group, and increased ReHo was discovered in the right thalamus, left inferior frontal gyrus and anterior subcallosal gyrus (Paakki et al. 2010). Another study showed lower activation in the superior parietal lobule and anterior prefrontal regions in the ASD group than in the TD group, and larger effects in the ASD group were detected in the lateral and medial temporal regions (Shukla et al. 2010). Maximo found overconnectivity in the ASD group in occipital and posterior temporal regions and underconnectivity in middle cingulate, posterior cingulate, and medial prefrontal regions (Maximo et al. 2013). Additionally, increased atypical functional connectivity was found in adolescents with ASD in bilateral temporo-occipital regions, and posterior overconnectivity was found to be associated with higher ASD symptom severity (Keown et al. 2013). Another facial expression fMRI task revealed that in the ASD group, connectivity in the middle temporal gyrus, amygdala, medial prefrontal gyrus, and inferior frontal gyrus was reduced (Sato et al. 2012). In summary, all of the abovementioned ASD studies identified specific brain areas involved in atypical social cognitive and emotion recognition, but their results were broad and contradictory. In this study, we wanted to find which brain area plays a pivotal role in negative emotion in individuals with autistic traits using functional and anatomical connectivity measures and examine this biomarker from a relative perspective.

Previous studies have investigated negative emotions in individuals with ASD or autistic traits in different task-based fMRI paradigms (Monk 2010; Koshino et al. 2008; Kliemann et al. 2018; lkeda et al. 2018). Task $\mathrm{fMRI}$ has been widely used in studies of neural basis of perception, cognition, and emotion studies (Hirata et al. 2018; Ciaramidaro et al. 2018; Sebastian 2015). Previous studies have traditionally focused on task-related fMRI to show increased neural connectivity, for instance, higher activity was found in an experimental state than in a baseline state (Van Eylen et al. 2018); this task fMRI study revealed individuals with autistic traits who were in the brain shifting mode and who demonstrated some task failure or poor performance related to specific cognitive function deficits. In Deruelle's emotion picture recognition study, negative emotion did not enhance recall skills in adults with ASD, which points to a 
reduced influence of emotion on memory process in ASD than in typically developing individuals (Deruelle et al. 2008; Kliemann et al. 2018). Hirata's social and nonsocial tasks study revealed significantly reduced brain activation in frontotemporal areas during both tasks in patients with ASD compared to healthy subjects (Hirata et al. 2018). Another task-based fMRI study using inhibitory tasks with eye gaze in ASD patients revealed that prefrontal areas may be affected by atypical gaze processing of socially relevant information in ASD (Ikeda et al. 2018). In a facial expression task fMRI study that found increased inferior frontal gyrus activity with age in subjects with autism compared to that in control subjects, the age-related increase in activity was associated with changes in gaze behavior and improvements in social functioningBastiaansen et al. 2011). The activation of the fusiform gyrus and amygdala was strongly positively correlated with time spent fixating on the eyes in the autistic and typically developing groups (Dalton et al. 2005). Above all, these studies focused on simple or standard faces and emotion types and generated negative feelings. In this study, we use extremely negative images to arouse participants' negative emotions to explore specific brain functions in individuals with autistic traits.

In the present study, the autism spectrum quotient (AQ) was used to measure individual levels of autistic traits, as well as to explore the neural basis of negative emotional processing in individuals with autistic traits in general young adults through a negative emotion processing task. Furthermore, we explored what key roles these atypical brain regions play in autistic traits and negative emotion processing. We hypothesize that in individuals with autistic traits, due to a high level of negative emotion and a relative insensitivity toward negative information compared to typically developing individuals, the middle temporal gyrus (MTG), which is responsible for deducing social information, may dominate the leading role in negative emotion processing within this group of people.

\section{Methods}

\section{Participants}

A total of 188 people were recruited for this study. Due to excessive head movements, 26 participants were excluded ( $>2.5 \mathrm{~mm}$ translation and angular rotation in each axis). Thus, a total of 162 participants participated in this study. Due to the nature of longitudinal data acquisition, these participants were familiar with the fMRI environment and paid little attention to it. In addition, 104 subjects with task fMRI results matched to the AQ ( 25 men, aged $18-25$ years, mean $=21.26$ years, standard deviation $=1.26$ ) participated in this research as part of our project investigating associations among genes, brain imaging and mental health (Liu et al. 2017). All methods were in accordance with the Declaration of Helsinki (W. M. Association 1991). All participants were right-handed, with no history of neurological or psychiatric problems. All participants provided written informed consent and received payment for their time. The study was approved by the Southwest University Brain Imaging Center Institutional Review Board.

\section{Assessment of the AQ}


The $A Q$ was used to measure autistic traits in individuals from the general population (S. Baron-Cohen et al. 2001). The Chinese version of the AQ (Lau et al. 2013) was used in this study, and consists of the Social Skill, Communication, Attention Switching, Imagination and Attention to Detail subscales, as represented by fifty statements; participants responded to these statements on a 4-point Likert scale, with "definitely agree" and "slightly agree" responses being scored one point and "slightly disagree" and "definitely disagree" responses being scored one point in the reverse-scored options. In half of the statements, the diagnostic answer is "agree", and in the other half, it is "disagree". One point is awarded for each diagnostic answer, which results in a continuous distribution of scores in the population sample. The total score ranges from 0 to 50 points, with higher scores suggesting a higher magnitude of autistic traits. Currently available data from research on the properties of this scale indicate that the measurement reliability for the total score is satisfactory (Austin 2005; Hoekstra et al. 2008; Ingersoll et al. 2011; Hurst et al. 2007; Kloosterman et al. 2011). In the present study we focused on analyzing the total $\mathrm{AQ}$ score.

\section{Negative emotion processing task}

In this study, a negative emotion processing task that has been used in many previous studies was adapted from established paradigms (Klemanski et al. 2017; Gross 1998; Paschke et al. 2016; Buruck et al. 2016). There were three conditions in this experiment, including 'watch neutral' (WatchNeu), 'watch negative' (WatchNeg) and 'decrease negative' (RegulateNeg) conditions. In this study, we focused only on the difference between WatchNeg and WatchNeu conditions and on the condition of WatchNegWatchNeu, which was termed negative emotion in the present study. In the 'WatchNeu' and 'WatchNeg" conditions, participants were told to watch neutral inanimate pictures (e.g., a bowl, an umbrella, etc.) or negative pictures (e.g., a bloody person in a car accident, a rotten corpse, etc.), which had all been viewed in a practice session before the test in the scanner.

In each trial, participants were given instructions $(2000 \mathrm{~ms})$ to watch a picture passively ('WatchNeu' or 'WatchNeg') during its presentation $(7000 \mathrm{~ms})$. Then, participants were asked to rate their emotions on a 5-point Likert scale (from ' 1 ' 'neutral', to ' 5 ' 'strongly negative') when the next slide (4500 ms) was presented. A fixation cross slide with a +" symbol $(2000 \mathrm{~ms})$ was used to separate each trial. The pictures used in this experiment were obtained from the International Affective Picture System (IAPS) database (Lang et al. 1997). The duration of the task was $11 \mathrm{~min}, 28 \mathrm{~s}$. The instructions, the presentation of a picture, a self-report rating and a fixation cross were included in each trial (see Fig. 1a).

\section{MRI Data Acquisition and Preprocessing}

All subjects underwent MRI scanning at the Brain Imaging Center of Southwest University. Whole-brain imaging data were obtained with a 3.0-T Siemens Trio MRI scanner with a 12-channel whole-brain coil, functional images including 32 continuous slices were obtained with a T2*-weighted (Siemens Medical, Erlangen, Germany). For each subject, 344 Blood Oxygen Level-Dependent (BOLD) images were acquired 
during the $\mathrm{fMRI}$ task with a gradient echo type echo planar imaging $(\mathrm{EPI})$ sequence [echo time $(\mathrm{TE})=30$ $\mathrm{ms}$; repetition time $(T R)=2000 \mathrm{~ms}$; flip angle $=90$ degrees; slice thickness $=3.0 \mathrm{~mm}$; slices $=32$; resolution matrix $=64 * 64$; voxel size $=3.4 * 3.4 * 4 \mathrm{~mm}$; field of view $($ FOV $)=220 \times 220 \mathrm{~mm}^{2}$; resolution matrix $=96 \times 96$; thickness $/$ inter slice gap $=3 / 1 \mathrm{~mm}$; and sampling bandwidth $=250 \mathrm{kHz}]$.

Then, a magnetization-prepared rapid gradient echo (MPRAGE) sequence was used to collected a highresolution anatomical image. This sequence was acquired with the following parameters: scan time $=4$ $\min 58 \mathrm{~s} ;$ slices $=176 ;$ slice thickness $=1.0 \mathrm{~mm} ; \mathrm{TE}=2.52 \mathrm{~ms} ; \mathrm{TR}=1900 \mathrm{~ms} ; \mathrm{TI}=900 \mathrm{~ms} ;$ sampling bandwidth $=31.2 \mathrm{kHz}$; flip angle $=9^{\circ}$; resolution matrix $=256 \times 256$; voxel size $=1 \times 1 \times 1 \mathrm{~mm}$; Preprocessing was performed by Data Processing Assistant for Resting-state fMRI (DPARSF; http://rfmri.org/ DPARSF) (Yan and Zang 2010), which is a toolbox based on the SPM8 software package (www.fil.ion.ucl.ac/spm). The functional data were realigned and unwrapped to correct head movement artifacts based on field maps. Whole images underwent temporal processing and slice timing correction, motion correction to reduce displacement between volumes, spatial normalization to the standard Montreal Neurological Institute (MNI) space with a resampled voxel size of $3 \times 3 \times 3 \mathrm{~mm}^{3}$ using the T1 scans as reference images, and spatial smoothing ( $6 \mathrm{~mm}$ full width half maximum, FWHM), band-pass temporal filtering $(0.01-0.1 \mathrm{~Hz})$. To minimize the effects of head motion, subjects with $>10 \%$ displaced frames, or maximal motion between volumes in each direction $>3 \mathrm{~mm}$, and rotation about each axis $>3^{\circ}$.

\section{Statistical Analysis}

\section{Task-based fMRI analyses}

In the first-level analysis, the general linear model (GLM) was used to separately estimate the effects in the two conditions (WatchNeu, WatchNeg), which were modeled by boxcar functions (picture $=7 \mathrm{~s}$, fixation $=2 \mathrm{~s}$ ) convolved with a canonical hemodynamic response function (HRF). In addition, six head motion parameters were taken as confounds. Then, we calculated 'contrast images' for each participant for the negative feeling perception WatchNeg > WatchNeu and computed the whole-brain activity pattern for this contrast, through which subject-specific effects were estimated by using the linear contrasts obtained from the first-level analysis. All fMRI data analyses were implemented in SPM8 (WhitfieldGabrieli and Nieto-Castanon 2012). Based on the abovementioned analysis, we then applied multiple linear regression analysis at the second level to obtain clusterwise statistics and to explore specific brain areas in which BOLD signal activity was associated with individuals with autistic traits, as tested by the $A Q$, within SPM8. To remove potential confounds, we used age and gender as nuisance covariates. The statistical threshold was set at $\mathrm{P}<0.05$, FWE (familywise error) corrected.

\section{Mediation analysis}


A mediation analysis was performed to test how activity in specific brain areas can play a pivotal role on the relationship between the $\mathrm{AQ}$ score and the WatchNeg-WatchNeu (negative emotion) condition score. We used PROCESS 3.3 (Hayes 2012) to conduct mediation analyses, and age and gender parameters were incorporated as covariates of no interest. The indirect mediation effect was estimated using bootstrapped sampling. We drew 5,000 bootstrapped samples, and a bias-corrected $95 \%$ bootstrap confidence interval that did not include zero indicated a significant indirect effect through the mediator (Preacher and Hayes 2008).

\section{Generalized psychophysiological interaction analyses (gPPI analyses)}

To further test the importance of the MTG in negative emotions in individuals with autistic traits, in the present study, we applied gPPI analyses with the CONN toolbox using preprocessed data (WhitfieldGabrieli and Nieto-Castanon 2012). In the first-level analysis, the seeds of the BOLD time course were extracted from each participant and condition, and then, a seed-to-voxel beta map was calculated, and the interaction between the seed region BOLD time series and the WatchNeg > WatchNeu contrast condition was computed. We used a seed-to-voxel approach to conduct gPPI analyses on the WatchNeg > WatchNeu condition. A familywise error (FWE)-corrected $p<0.05$ at the cluster level and an uncorrected $p$ $<0.001$ at the voxel level were adopted in this study. Cerebrospinal fluid (CSF), white matter, ART-based scrubbing, and 6 rigid-body parameters were regressed out of whole-brain gray matter activity. To reduce the effect of low-frequency drift and high-frequency noise, bandpass filtering $(0.01<f<0.1 \mathrm{~Hz})$ was used. The peak activation in the right MTG (MNl: 48, -18, -18) was used as the seed from the multiple linear regression results that were activated in the WatchNeg $>$ WatchNeu contrast. We included age and sex as covariates in the multiple regression analysis.

\section{Results}

\section{Descriptive statistics}

The demographic data and behavioral results are shown in Tab. 1. The mean AQ score of the current sample was 19.78, and the standard deviation was 5.29 .

\section{Tab. 1}

\section{Negative emotion processing task performance}

We calculated the mean values of the negative feelings ratings in WatchNeg and WatchNeu conditions. The results of a pared-samples t-test showed that the ratings of negative feelings were significantly 
higher in the WatchNeg condition $(M \pm S D=3.35 \pm 0.70)$ than in the WatchNeu condition $(M \pm S D$ $=1.22 \pm 0.38, t=32.62, p<0.001$ ) (Fig. 1b)

Fig. 1a, 1b

\section{Brain activation between WatchNeg-WatchNeu condition and AQ (total) score}

Activation during WatchNeg-WatchNeu condition showed increased activation in the right middle temporal gyrus (MTG) (peak MNI coordinate $=48,-18,-18, T=5.12$, cluster size: $33 ; p<0.05$, FWEcorrected), and hippocampal gyrus (HG) (peak MNI coordinate $=21,-12,-18, T=3.76$, cluster size: $11 ; p<$ 0.001 uncorrected). Extracted beta values from these two brain areas were used for Pearson correlation analysis with the $A Q$ (total) score. The results showed that the $A Q$ (total) score was positively associated with activation in the right MTG $(r=0.438, p<0.0001)$ and right HG $(r=0.362, p<0.0001)$, as shown in Fig.2.

\section{Fig.2.}

\section{Mediation results}

The mediation analysis found that the right MTG suppressed the relationship between the AQ (total) score and negative emotion. In the present study, the AQ (total) score was significantly positively associated with the right MTG $(r=.4406, p<.0001)$. The right MTG was significantly positively related to the negative emotion score $(r=.2176, p<0.05)$. The negative emotion score was negatively related to the AQ (total) score $\left(r=-.1150, p=.2564\right.$, nonsignificant). If $c^{\prime}$ had the opposite sign to that of ab, referred to by (MacKinnon et al. 2007) as inconsistent mediation, the absolute value of ab\c' would be 0.84 . Then, we used bootstrap resampling to test the significance of the indirect effect between the AQ (total) and negative emotion. A suppressing effect was found between the $\mathrm{AQ}$ and negative emotion, $\mathrm{Cl}[0.0006$, 0.0231, respectively], through the activation of the right MTG, see Fig. $\mathbf{3}$.

\section{Fig. 3}


To examine the functional connectivity between the $A Q$ and negative emotion processing, we implemented gPPI analyses with the right MTG as the seed from multiple linear regression results in the contrast of WatchNeg-WatchNeu. After controlling for the effects of age and sex, multiple regression analysis revealed that the $\mathrm{AQ}$ (total) score was related to higher functional connectivity between the right MTG and left parahippocampal gyrus (PHG) (peak MNI coordinate $=-30,-18,-30, T=4.46$, cluster size $=$ 66 , p-uncorrected $<0.001$ ).

\section{Discussion}

In the present study, we focused on the role MTG plays in negative emotion processing in individuals with autistic traits using a task-based fMRI paradigm examining emotion regulation in a large sample confirmed by AQ. We focused on WatchNeg and WatchNeu conditions because of our research theme. The results show that the mean values of the negative feelings ratings differences in the WatchNeg and WatchNeu conditions were significant. When individuals with autistic traits watch negative events, the right MTG is significantly activated relative to its activation when individuals watch neural events. More importantly, the mediation analysis showed that the right MTG fully mediates the association between the AQ (total) score and negative emotional processing, especially between the communication subscale of $A Q$ and negative emotional processing, suggesting that people with autistic traits might demonstrate altered negative emotion processing through the right MTG. Furthermore, gPPI analysis also suggested that the right MTG is significantly functionally connected (FC) with the left parahippocampus gyrus $(\mathrm{PHG})$. Taken together, we concluded that the right MTG plays a pivotal role in the processing of negative emotions in individuals with autistic traits.

First, the MTG is responsible for various social and communicative functions (Avidan et al. 2014; Cohen Kadosh and Johnson 2007; Deeley et al. 2007; Pelphrey et al. 2004; Sugiura et al. 2014; von dem Hagen et al. 2014; Kitada et al. 2010; Plaut and Behrmann 2013; Schilbach 2015). In a face perception study, individuals with congenital prosopagnosiashowed reduced activation and connectivity in the anterior temporal cortexAvidan et al. 2014). Another study claimed both word and face recognition rely on finegained visual representations, by virtue of pressure to couple visual and language areas and keep to connection length short (Plaut and Behrmann 2013). Cohen also claims that face processing in the adult brain involves the fusiform gyrus, superior temporal sulcus and occipito-temporal cortex (Cohen Kadosh and Johnson 2007). In studies on identification of facial expression in emotion, standard facial expressions such as disgust, neutrallty, and happiness are related to MTG, IPL, and IFG et al, in this study, these areas are activated by haptic rather than visual identification (Kitada et al. 2010). In the detection and processing of social cues, the right posterior superior temporal gyrus thought to be involved in analyzing the intentions of other people's actions, and activity in this region is sensitive to the context of the observed biological motions (Pelphrey et al. 2004). In another social signal study, participant variation in neural responses in the right posterior MTG during implicit eye-gaze detection suggesed the individual's tendency to recruit regions related to mental-state inference during the implicit mental inference (IMI) task, which suggests that the implicit detection of visual social signals involves the PMTG and underlies the development of higher social cognition (Sugiura et al. 2014). All of these studies involved the MTG 
either due to clinical patients with obvious deficits or other experimental conditions, such as haptic identification or mentalism. In the present study, when negative emotions were aroused in people with autistic traits by watching negative images, the right MTG was activated. This phenomenon occurred because individuals with autistic traits may have deficits in avoiding negative situations, and they are tend to deduce what happened to the people or the object in the image rather than react to image itself.

Second, we also found that the right MTG has a suppressing effect on the relationship between the AQ (total) score and negative emotion. Emotion-processing difficulties appear to be a hallmark feature of ASD, and most studies on autism have come to the consensus that individuals with autistic traits have general deficits in processing negative emotion modalities and affective stimuli originating (Ciaramidaro et al. 2018; Deruelle et al. 2008; Klemanski et al. 2017; Santos et al. 2012). In Ciaramidaro's study, ASD patient impairments seem to be more pronounced during implicit rather than explicit negative facial affect recognition (Ciaramidaro et al. 2018). In addition to negative emotions, social anxiety and depression are also common mental health problems among young adults, in addition, repetitive negative thinking may lead to anxiety and mood disorders in young adults (Klemanski et al. 2017). In Santos's study, the ASD group failed to show a bias toward threat-related scenes, which means ASD patients were unaffected by threat scenes (Santos et al. 2012). Another study also claimed a reduced influence of emotion in ASD than in typical developing individuals (Deruelle et al. 2008). The above mentioned studies indicate that individuals with autistic traits whose level of negative emotion was lower than that of normal people, which confirming our hypothesis that the higher $A Q$ score is, the stronger the activation of the MTG, and the smaller distinction between WatchNeg and WatchNeu conditions. That is, when people with autistic traits perform negative emotion processing tasks, they prefer to deduce "what happened" rather than "what is" in the picture scene, and thus the impact of the negative images on them is reduced to some extent.

Third, gPPI analysis showed that the right MTG has a increased FC with the left PHG rather than the amygdala. The brain functions of the PHG are responsible for unconscious relational encoding (Duss et al. 2014; Tanweer et al. 2010). The FC between the MTG and PHG suggested that individuals with autistic traits process negative emotion unconsciously when performing a negative eomtion processing fMRI task. In previous studies, it has been thoroughly shown that ASD patients have an amygdala dysfunction (Ashwin et al. 2007; S. Baron-Cohen,. et al. 2000; Guo et al. 2016; Johns et al. 2019; Van Reekum et al. 2007). In Ashwin's study, higher activation was observed in the left amygdala and orbito-frontal cortex in control subjects, while the ASD group showed higher activation in the anterior cingulate and superior temporal gyrus (Ashwin et al. 2007). Reekum's study also showed relative to neutral information faster judgments of negative were associated with increased left and right anygdala activation (Van Reekum et al. 2007). Another study demonstrated that the amygdala is one of several brain regions that is abnormal in autism (S. Baron-Cohen,. et al. 2000). Guo's study also supported that adolescents with autism showed decreased FC between the amygdala and subcortical regions (Guo et al. 2016). Meanwhile, increased social impairment was found in preterm-born adolescents compared to their term-born peers, and amygdala connectivity was altered in those prematurely-born, markers of social functioning were correlated with altered amygdala-PCC connectivity (Johns et al. 2019). In our study, individuals with 
autistic traits showed FC between the right MTG and the left PHG rather than the amygdala, which is consistent with the results of previous studiest. Individuals with high a high AQ score who eaperience an atypical level of negative emotion and who are unsensitive to nagetive information (Ciaramidaro et al. 2018), deduced of negative information rather than evaluated (Pelphrey et al. 2004; Sugiura et al. 2014), tending to encode negative information unconsciously (Duss et al. 2014). The gPPI analysis further demonstrated the right MTG is a biomarker in individuals with autistic traits people coping encounter negative information.

\section{Limilations}

The current study investigate individuals with autistic traits which area of the brain play a pivotal role in processing negative emtion fMRI task. In paiticular, our autistic traits sample are all university students, which meas thay all passed the strict exaination and got a well score, thay all showing above-average intellence and no language deficits, there may have a bias in the sample compared to sub-average. In our sample there is no differences between the gender, in the future study, we will broden the sample size and take the gender into account. Liskewise, in the present study, we select two of three conditions as our focused theme, we want to ues a more specific task to explore negative emotion in individuals with autistic traits in the future study.

\section{Conclusion}

In conclusion, the present study used a negative emotion processing task fMRI experiment to explore which brain areas play a pivotal role in negative emotion in individuals with autistic traits. The results showed that the right MTG is an important brain region in people with autistic traits. We also provide potential evidence that negative and neutral emotions are processed more similarly in people with high levels of autistic traits than in typically developing people because of the altered development of the MTG. Furthermore, we provide insight into the task fMRI study specificity of autistic traits in a large sample. Moreover, our study provides a wider discussion about autism brain activation and functional connectivity patterns and the use the MTG as a biomarker in individuals with autistic traits.

\section{Abbreviations}

ASD: Autism spectrum disorder

AQ: Autism spectrum Quotient

FC: Functional connectivity

GLM: General linear model

fMRI: functional Magnetic Resonance Imaging 
MTG: Middle temporal gyrus

HG: Hippocampus gyrus

PHG: Parahippocampal gyrus

gPPI: generalized psychophysiological interaction analyses

\section{Declarations}

\section{Ethics approval and consent to participate}

All methods were in accordance with the Declaration of Helsinki. All participants provided written informed consent and received payment for their time. The study was approved by the Southwest University Brain Imaging Center Institutional Review Board.

\section{Consent for publication}

No application

\section{Availability of data and materials}

Please contact me feel free when you interested in our data.

Email: yu0071@email.swu.edu.cn

\section{Funding}

This research was supported by the National Natural Science Foundation of China $(31470981 ; 31571137$; $31500885 ; 31600878 ; 31771231)$, Natural Science Foundation of Chongqing (cstc2015jcyjA10106), Fok Ying Tung Education Foundation (151023).

\section{Competing interests}

The authors declared no competing interests.

\section{Author contributions}

Qiu conceived the experiments, Yu and Wang conducted the experiments, analyzed the results and carried on paper writing, Jamie provided the $A Q$ questionnaire and proposed many constructive advises in manuscript. All authors reviewed the manuscript.

Yaxu Yu and Xiaoqin Wang contributed equally to this work. Qiu is the corresponding author.

\section{Acknowledgements}


We thank all the participants who take part in this study. We also thank our Professor Qiu provide ideas, modification and spiritual help.

\section{Authors details}

${ }^{1}$ School of Psychology, Southwest University, Chongqing, China

${ }^{2}$ Key Laboratory of Cognition and Personality, Ministry of Education, Chongqing, China

${ }^{3}$ School of Psychology and ${ }^{4}$ Sackler Center for Consciousness Science, University of Sueesx, Brighton, BN1 9QH United Kingdom

*Corresponding Author. qiuj318@swu.edu.cn

+These authors have contributed equally to this work

Telephone numbers: 86-13368452014

\section{References}

Alaerts, K., Woolley, D. G., Steyaert, J., Di Martino, A., Swinnen, S. P., \& Wenderoth, N. (2014).

Underconnectivity of the superior temporal sulcus predicts emotion recognition deficits in autism. Soc Cogn Affect Neurosci, 9(10), 1589-1600, doi:10.1093/scan/nst156.

Ashwin, C., Baron-Cohen, S., Wheelwright, S., O’Riordan, M., \& Bullmore, E. T. (2007). Differential activation of the amygdala and the 'social brain' during fearful face-processing in Asperger Syndrome.

Neuropsychologia, 45(1), 2-14, doi:10.1016/j.neuropsychologia.2006.04.014.

Association, A. P. (2013). Diagnostic and statistical manual of mental disorders (DSM-5®): American Psychiatric Pub.

Association, W. M. (1991). Declaration of Helsinki. Law, medicine \& health care: a publication of the Amercian Society of Law \& Medicine. Gastroenterologia Japonica, 26(2), 269-270.

Austin, E. J. (2005). Personality correlates of the broader autism phenotype as assessed by the Autism Spectrum Quotient (AQ). Personality and Individual Differences, 38(2), 451-460.

Avidan, G., Tanzer, M., Hadj-Bouziane, F., Liu, N., Ungerleider, L. G., \& Behrmann, M. (2014). Selective dissociation between core and extended regions of the face processing network in congenital prosopagnosia. Cereb Cortex, 24(6), 1565-1578, doi:10.1093/cercor/bht007.

Baron-Cohen, S.,., Ring, H. A., Bullmore, E. T., Wheelwright, S.,., Ashwin, C.,., \& Williams, S. C. (2000). The amygdala theory of autism. Neuroscience \& Biobehavioral Reviews, 24(3), 355-364. 
Baron-Cohen, S., Wheelwright, S., Skinner, R., Martin, J., \& Clubley, a. E. (2001). The Autism-Spectrum Quotient (AQ): Evidence from Asperger Syndrome/High-Functioning Autism, Males and Females, Scientists and Mathematicians. Journal of Autism and Developmental Disorders, 31(1), 13.

Barrio, V. (2017). Diagnostic and Statistical Manual of Mental Disorders. In.

Barros-Loscertales, A., Bustamante, J. C., Ventura-Campos, N., Llopis, J. J., Parcet, M. A., \& Avila, C. (2011). Lower activation in the right frontoparietal network during a counting Stroop task in a cocainedependent group. Psychiatry Res, 194(2), 111-118, doi:10.1016/j.pscychresns.2011.05.001.

Bastiaansen, J. A., Thioux, M., Nanetti, L., van der Gaag, C., Ketelaars, C., Minderaa, R., et al. (2011). Agerelated increase in inferior frontal gyrus activity and social functioning in autism spectrum disorder. Biol Psychiatry, 69(9), 832-838, doi:10.1016/j.biopsych.2010.11.007.

Bird, G., \& Cook, R. (2013). Mixed emotions: the contribution of alexithymia to the emotional symptoms of autism. Transl Psychiatry, 3, e285, doi:10.1038/tp.2013.61.

Buruck, G., Dorfel, D., Kugler, J., \& Brom, S. S. (2016). Enhancing well-being at work: The role of emotion regulation skills as personal resources. J Occup Health Psychol, 21(4), 480-493, doi:10.1037/ocp0000023.

Carper, R. A., \& Courchesne, E. (2005). Localized enlargement of the frontal cortex in early autism. Biological psychiatry, 57(2), 126-133.

Ciaramidaro, A., Bolte, S., Schlitt, S., Hainz, D., Poustka, F., Weber, B., et al. (2018). Transdiagnostic deviant facial recognition for implicit negative emotion in autism and schizophrenia. Eur Neuropsychopharmacol, 28(2), 264-275, doi:10.1016/j.euroneuro.2017.12.005.

Cohen Kadosh, K., \& Johnson, M. H. (2007). Developing a cortex specialized for face perception. Trends Cogn Sci, 11(9), 367-369, doi:10.1016/j.tics.2007.06.007.

Constantino, J. N., \& Todd, R. D. (2003). Autistic Traits in the General Population: A Twin Study. Jama Psychiatry, 60(5), 524-530, doi:10.1001/archpsyc.60.5.524.

Constantino, J. N., \& Todd, R. D. (2005). Intergenerational transmission of subthreshold autistic traits in the general population. Biological psychiatry, 57(6), 655-660.

Dalton, K. M., Nacewicz, B. M., Johnstone, T., Schaefer, H. S., Gernsbacher, M. A., Goldsmith, H. H., et al. (2005). Gaze fixation and the neural circuitry of face processing in autism. Nat Neurosci, 8(4), 519-526, doi:10.1038/nn1421.

Damarla, S. R., Keller, T. A., Kana, R. K., Cherkassky, V. L., Williams, D. L., Minshew, N. J., et al. (2010). Cortical underconnectivity coupled with preserved visuospatial cognition in autism: Evidence from an fMRI study of an embedded figures task. Autism Res, 3(5), 273-279, doi:10.1002/aur.153. 
Dawson, G., Toth, K., Abbott, R., Osterling, J., Munson, J., Estes, A., et al. (2004). Early social attention impairments in autism: social orienting, joint attention, and attention to distress. Developmental Psychology, 40(2), 271.

Deeley, Q., Daly, E. M., Surguladze, S., Page, L., Toal, F., Robertson, D., et al. (2007). An event related functional magnetic resonance imaging study of facial emotion processing in Asperger syndrome. Biol Psychiatry, 62(3), 207-217, doi:10.1016/j.biopsych.2006.09.037.

Deruelle, C., Hubert, B., Santos, A., \& Wicker, B. (2008). Negative emotion does not enhance recall skills in adults with autistic spectrum disorders. Autism Res, 1(2), 91-96, doi:10.1002/aur.13.

Duss, S. B., Reber, T. P., Hanggi, J., Schwab, S., Wiest, R., Muri, R. M., et al. (2014). Unconscious relational encoding depends on hippocampus. Brain, 137(Pt 12), 3355-3370, doi:10.1093/brain/awu270.

Eack, S. M., Mazefsky, C. A., \& Minshew, N. J. (2015). Misinterpretation of facial expressions of emotion in verbal adults with autism spectrum disorder. Autism, 19(3), 308-315, doi:10.1177/1362361314520755.

Gotts, S. J., Simmons, W. K., Milbury, L. A., Wallace, G. L., Cox, R. W., \& Martin, A. (2012). Fractionation of social brain circuits in autism spectrum disorders. Brain, 135(Pt 9), 2711-2725, doi:10.1093/brain/aws160.

Grootscholten, I. A. C., van Wijngaarden, B., \& Kan, C. C. (2018). High Functioning Autism Spectrum Disorders in Adults: Consequences for Primary Caregivers Compared to Schizophrenia and Depression. $J$ Autism Dev Disord, doi:10.1007/s10803-017-3445-1.

Gross, J. J. (1998). Antecedent-and response-focused emotion regulation: divergent consequences for experience, expression, and physiology. J Pers Soc Psychol, 74(1), 224.

Guo, X., Duan, X., Long, Z., Chen, H., Wang, Y., Zheng, J., et al. (2016). Decreased amygdala functional connectivity in adolescents with autism: A resting-state fMRI study. Psychiatry Res Neuroimaging, 257, 47-56, doi:10.1016/j.pscychresns.2016.10.005.

Hayes, A. F. (2012). PROCESS: A versatile computational tool for observed variable mediation, moderation, and conditional process modeling. University of Kansas, KS.

Hirata, K., Egashira, K., Harada, K., Nakashima, M., Hirotsu, M., Isomura, S., et al. (2018). Differences in frontotemporal dysfunction during social and non-social cognition tasks between patients with autism spectrum disorder and schizophrenia. Sci Rep, 8(1), 3014, doi:10.1038/s41598-018-21379-w.

Hoekstra, R. A., Bartels, M., Cath, D.C., \& Boomsma, D. I. (2008). Factor structure, reliability and criterion validity of the Autism-Spectrum Quotient (AQ): a study in Dutch population and patient groups. $J$ Autism Dev Disord, 38(8), 1555-1566, doi:10.1007/s10803-008-0538-x. 
Hurst, R. M., Mitchell, J. T., Kimbrel, N. A., Kwapil, T. K., \& Nelson-Gray, R. O. (2007). Examination of the reliability and factor structure of the Autism Spectrum Quotient (AQ) in a non-clinical sample. Personality and Individual Differences, 43(7), 1938-1949, doi:10.1016/j.paid.2007.06.012.

Ikeda, T., Hirai, M., Sakurada, T., Monden, Y., Tokuda, T., Nagashima, M., et al. (2018). Atypical neural modulation in the right prefrontal cortex during an inhibitory task with eye gaze in autism spectrum disorder as revealed by functional near-infrared spectroscopy. Neurophotonics, 5(3), 035008, doi:10.1117/1.NPh.5.3.035008.

Ingersoll, B., Hopwood, C. J., Wainer, A., \& Brent Donnellan, M. (2011). A comparison of three self-report measures of the broader autism phenotype in a non-clinical sample. J Autism Dev Disord, 41(12), 16461657, doi:10.1007/s10803-011-1192-2.

Jansen, P. R., Polderman, T. J. C., Bolhuis, K., van der Ende, J., Jaddoe, V. W. V., Verhulst, F. C., et al. (2018). Polygenic scores for schizophrenia and educational attainment are associated with behavioural problems in early childhood in the general population. Journal of Child Psychology and Psychiatry, 59(1), 39-47, doi:10.1111/jcpp.12759.

Johns, C. B., Lacadie, C., Vohr, B., Ment, L. R., \& Scheinost, D. (2019). Amygdala functional connectivity is associated with social impairments in preterm born young adults. Neuroimage Clin, 21, 101626, doi:10.1016/j.nicl.2018.101626.

Katarzyna, C., Fred, V., \& Ami, K. (2010). Limited attentional bias for faces in toddlers with autism spectrum disorders. Archives of general psychiatry, 67(2), 178-185.

Keown, C. L., Shih, P., Nair, A., Peterson, N., Mulvey, M. E., \& Muller, R. A. (2013). Local functional overconnectivity in posterior brain regions is associated with symptom severity in autism spectrum disorders. Cell Rep, 5(3), 567-572, doi:10.1016/j.celrep.2013.10.003.

Kitada, R., Johnsrude, I. S., Kochiyama, T., \& Lederman, S. J. (2010). Brain networks involved in haptic and visual identification of facial expressions of emotion: an fMRI study. Neuroimage, 49(2), 1677-1689, doi:10.1016/j.neuroimage.2009.09.014.

Klemanski, D. H., Curtiss, J., McLaughlin, K. A., \& Nolen-Hoeksema, S. (2017). Emotion Regulation and the Transdiagnostic Role of Repetitive Negative Thinking in Adolescents with Social Anxiety and Depression. Cognit Ther Res, 41(2), 206-219, doi:10.1007/s10608-016-9817-6.

Kliemann, D., Richardson, H., Anzellotti, S., Ayyash, D., Haskins, A. J., Gabrieli, J. D. E., et al. (2018). Cortical responses to dynamic emotional facial expressions generalize across stimuli, and are sensitive to task-relevance, in adults with and without Autism. Cortex, 103, 24-43, doi:10.1016/j.cortex.2018.02.006. 
Kloosterman, P. H., Keefer, K. V., Kelley, E. A., Summerfeldt, L. J., \& Parker, J. D. (2011). Evaluation of the factor structure of the Autism-Spectrum Quotient. Personality and Individual Differences, 50(2), 310-314.

Koshino, H., Kana, R. K., Keller, T. A., Cherkassky, V. L., Minshew, N. J., \& Just, M. A. (2008). fMRI investigation of working memory for faces in autism: visual coding and underconnectivity with frontal areas. Cereb Cortex, 18(2), 289-300, doi:10.1093/cercor/bhm054.

Lang, P. J., Bradley, M. M., \& Cuthbert, B. N. (1997). International affective picture system (IAPS): Technical manual and affective ratings. NIMH Center for the Study of Emotion and Attention, 1, 39-58.

Lau, W. Y.-P., Gau, S. S.-F., Chiu, Y.-N., Wu, Y.-Y., Chou, W.-J., Liu, S.-K., et al. (2013). Psychometric properties of the Chinese version of the Autism Spectrum Quotient (AQ). Research in developmental disabilities, 34(1), 294-305.

Liu, W., Wei, D., Chen, Q., Yang, W., Meng, J., Wu, G., et al. (2017). Longitudinal test-retest neuroimaging data from healthy young adults in southwest China. Sci Data, 4, 170017, doi:10.1038/sdata.2017.17.

MacKinnon, D. P., Fairchild, A. J., \& Fritz, M. S. (2007). Mediation analysis. Annu Rev Psychol, 58, 593614, doi:10.1146/annurev.psych.58.110405.085542.

Maximo, J. O., Keown, C. L., Nair, A., \& Müller, R.-A. (2013). Approaches to local connectivity in autism using resting state functional connectivity MRI. Frontiers in human neuroscience, 7, doi:10.3389/fnhum.2013.00605.

Monk, C. (2010). Neural circuitry of emotional face processing in autism spectrum disorders. Journal of Psychiatry and Neuroscience, 35(2), 105-114, doi:10.1503/jpn.090085.

Mueller, S., Keeser, D., Samson, A. C., Kirsch, V., Blautzik, J., Grothe, M., et al. (2013). Convergent Findings of Altered Functional and Structural Brain Connectivity in Individuals with High Functioning Autism: A Multimodal MRI Study. PLoS One, 8(6), e67329, doi:10.1371/journal.pone.0067329.

Muskett, A., Capriola-Hall, N. N., Radtke, S. R., Factor, R., \& Scarpa, A. (2019). Repetitive behaviors in Autism Spectrum Disorder: Associations with depression and anxiety symptoms. Research in Autism Spectrum Disorders, 68, 101449, doi:10.1016/j.rasd.2019.101449.

New, J. J., Schultz, R. T., Wolf, J., Niehaus, J. L., Klin, A., German, T. C., et al. (2010). The scope of social attention deficits in autism: prioritized orienting to people and animals in static natural scenes. Neuropsychologia, 48(1), 51-59, doi:10.1016/j.neuropsychologia.2009.08.008.

Paakki, J. J., Rahko, J., Long, X., Moilanen, I., Tervonen, O., Nikkinen, J., et al. (2010). Alterations in regional homogeneity of resting-state brain activity in autism spectrum disorders. Brain Res, 1321, 169179, doi:10.1016/j.brainres.2009.12.081. 
Paschke, L. M., Dörfel, D., Steimke, R., Trempler, I., Magrabi, A., Ludwig, V. U., et al. (2016). Individual differences in self-reported self-control predict successful emotion regulation. Soc Cogn Affect Neurosci, 11(8), 1193-1204.

Pelphrey, K. A., Morris, J. P., \& McCarthy, G. (2004). Grasping the Intentions of Others: The Perceived Intentionality of an Action Influences Activity in the Superior Temporal Sulcus during Social Perception. Journal of Cognitive Neuroscience, 16(10), 1706-1716, doi:10.1162/0898929042947900.

Pierce, K., Conant, D., Hazin, R., Stoner, R., \& Desmond, J. (2011). Preference for geometric patterns early in life as a risk factor for autism. Archives of general psychiatry, 68(1), 101-109.

Plaut, D.C., \& Behrmann, M. (2013). Response to Susilo and Duchaine: beyond neuropsychological dissociations in understanding face and word representations. Trends Cogn Sci, 17(11), 546, doi:10.1016/j.tics.2013.09.010.

Preacher, K. J., \& Hayes, A. F. J. B. R. M. (2008). Asymptotic and resampling strategies for assessing and comparing indirect effects in multiple mediator models.

Redcay, E., \& Courchesne, E. (2005). When is the brain enlarged in autism? A meta-analysis of all brain size reports. Biol Psychiatry, 58(1), 1-9, doi:10.1016/j.biopsych.2005.03.026.

Samson, A. C., Hardan, A. Y., Lee, I. A., Phillips, J. M., \& Gross, J. J. (2015). Maladaptive Behavior in Autism Spectrum Disorder: The Role of Emotion Experience and Emotion Regulation. J Autism Dev Disord, 45(11), 3424-3432, doi:10.1007/s10803-015-2388-7.

Santos, A., Chaminade, T., Da Fonseca, D., Silva, C., Rosset, D., \& Deruelle, C. (2012). Just another social scene: evidence for decreased attention to negative social scenes in high-functioning autism. $J$ Autism Dev Disord, 42(9), 1790-1798, doi:10.1007/s10803-011-1415-6.

Sasamoto, A., Miyata, J., Hirao, K., Fujiwara, H., Kawada, R., Fujimoto, S., et al. (2011). Social impairment in schizophrenia revealed by Autism-Spectrum Quotient correlated with gray matter reduction. Soc Neurosci, 6(5-6), 548-558.

Sato, W., Toichi, M., Uono, S., \& Kochiyama, T. (2012). Impaired social brain network for processing dynamic facial expressions in autism spectrum disorders. BMC neuroscience, 13(1), 99.

Schilbach, L. (2015). Eye to eye, face to face and brain to brain: novel approaches to study the behavioral dynamics and neural mechanisms of social interactions. Current Opinion in Behavioral Sciences, 3, 130135, doi:10.1016/j.cobeha.2015.03.006.

Schipul, S. E., Williams, D. L., Keller, T. A., Minshew, N. J., \& Just, M. A. (2012). Distinctive neural processes during learning in autism. Cereb Cortex, 22(4), 937-950, doi:10.1093/cercor/bhr162. 
Sebastian, C. L. (2015). Social cognition in adolescence: Social rejection and theory of mind. Psicología Educativa, 21(2), 125-131, doi:10.1016/j.pse.2015.08.004.

Shukla, D. K., Keehn, B., \& Muller, R. A. (2010). Regional homogeneity of fMRI time series in autism spectrum disorders. Neurosci Lett, 476(1), 46-51, doi:10.1016/j.neulet.2010.03.080.

St Pourcain, B., Robinson, E. B., Anttila, V., Sullivan, B. B., Maller, J., Golding, J., et al. (2018). ASD and schizophrenia show distinct developmental profiles in common genetic overlap with population-based social communication difficulties. Molecular Psychiatry, 23(2), 263-270, doi:10.1038/mp.2016.198.

Sucksmith, E., Roth, I., \& Hoekstra, R. A. (2011). Autistic Traits Below the Clinical Threshold: Re-examining the Broader Autism Phenotype in the 21st Century. Neuropsychology Review, 21(4), 360-389, doi:10.1007/s11065-011-9183-9.

Sugiura, M., Yomogida, Y., Mano, Y., Sassa, Y., Kambara, T., Sekiguchi, A., et al. (2014). From social-signal detection to higher social cognition: an fMRI approach. Soc Cogn Affect Neurosci, 9(9), 1303-1309, doi:10.1093/scan/nst119.

Tanweer, T., Rathbone, C. J., \& Souchay, C. (2010). Autobiographical memory, autonoetic consciousness, and identity in Asperger syndrome. Neuropsychologia, 48(4), 900-908.

Tyszka, J. M., Kennedy, D. P., Paul, L. K., \& Adolphs, R. (2014). Largely typical patterns of resting-state functional connectivity in high-functioning adults with autism. Cereb Cortex, 24(7), 1894-1905, doi:10.1093/cercor/bht040.

Van Eylen, L., Boets, B., Steyaert, J., Wagemans, J., \& Noens, I. (2018). Local and global visual processing in autism spectrum disorders: Influence of task and sample characteristics and relation to symptom severity. Journal of Autism and Developmental Disorders, 48(4), 1359-1381.

Van Reekum, C. M., Urry, H. L., Johnstone, T., Thurow, M. E., Frye, C. J., Jackson, C. A., et al. (2007). Individual differences in amygdala and ventromedial prefrontal cortex activity are associated with evaluation speed and psychological well-being. Journal of Cognitive Neuroscience, 19(2), 237-248.

Vissers, M. E., Cohen, M. X., \& Geurts, H. M. (2012). Brain connectivity and high functioning autism: a promising path of research that needs refined models, methodological convergence, and stronger behavioral links. Neuroscience \& Biobehavioral Reviews, 36(1), 604-625.

von dem Hagen, E. A., Stoyanova, R. S., Baron-Cohen, S., \& Calder, A. J. (2013). Reduced functional connectivity within and between 'social' resting state networks in autism spectrum conditions. Soc Cogn Affect Neurosci, 8(6), 694-701, doi:10.1093/scan/nss053.

von dem Hagen, E. A., Stoyanova, R. S., Rowe, J. B., Baron-Cohen, S., \& Calder, A. J. (2014). Direct gaze elicits atypical activation of the theory-of-mind network in autism spectrum conditions. Cereb Cortex, 24(6), 1485-1492, doi:10.1093/cercor/bht003.

Page 19/20 
Wass, S. (2011). Distortions and disconnections: disrupted brain connectivity in autism. Brain and cognition, 75(1), 18-28.

Wheelwright, S., Auyeung, B., Allison, C., \& Baron-Cohen, S. (2010). Defining the broader, medium and narrow autism phenotype among parents using the Autism Spectrum Quotient (AQ). Molecular autism, 1(1), 10.

Whitfield-Gabrieli, S., \& Nieto-Castanon, A. (2012). Conn: a functional connectivity toolbox for correlated and anticorrelated brain networks. Brain Connect, 2(3), 125-141, doi:10.1089/brain.2012.0073.

Yan, C., \& Zang, Y. (2010). DPARSF: a MATLAB toolbox for "pipeline" data analysis of resting-state fMRI. [Methods]. Frontiers in Systems Neuroscience, 4(13), doi:10.3389/fnsys.2010.00013.

Zhu, H., Fan, Y., Guo, H., Huang, D., \& He, S. (2014). Reduced interhemispheric functional connectivity of children with autism spectrum disorder: evidence from functional near infrared spectroscopy studies. Biomed Opt Express, 5(4), 1262-1274, doi:10.1364/BOE.5.001262.

\section{Table}

Table1. A summary of the demographic information in the present study

\begin{tabular}{lccc}
\hline \multirow{2}{*}{ Measure } & \multicolumn{3}{c}{$\mathrm{N}=104$} \\
\cline { 2 - 4 } & Mean & SD & range \\
\hline Age & 21.26 & 1.26 & $18-25$ \\
AQ(total) & 19.78 & 5.29 & $9-31$ \\
\hline Social skill & 3.85 & 2.36 & $0-9$ \\
Attention switching & 5.31 & 1.82 & $1-10$ \\
\hline Attention to detail & 4.60 & 2.05 & $1-10$ \\
\hline Communication & 3.16 & 1.91 & $0-8$ \\
\hline Imagination & 2.87 & 1.56 & $0-6$ \\
\hline
\end{tabular}

Notes: $A Q=$ Autism Spectrum Quotient; $S D=$ standard deviation; $N=$ number 